\title{
Clinical Efficacy and Safety Evaluation of Ulinastatin Combined with Octreotide in the Treatment of Patients with Severe Acute Pancreatitis
}

\author{
Q. DING, YUXIA YAO ${ }^{1}$, HUIBO WU'1, BINBIN XIA AND H. CHENG*
}

Department of Pharmacy, 1Department of Digestive System, Beijing Luhe Hospital Captial Medical Univeisity, No. 82, Xinhua South Road, Tongzhou District, Beijing, China

\section{Ding et al.: Ulinastatin and Octreotide Combination in Patients with Severe Acute Pancreatitis}

\begin{abstract}
To investigate the therapeutic efficacy and safety of the combination of ulinastatin and octreotide in the treatment of patients with severe acute pancreatitis. A total of 224 acute pancreatitis patients were selected in our hospital from January 2017 to December 2019 as the subjects of this retrospective study. Among them, observation group $(n=112)$ and control group $(n=112)$. Both groups were treated with conventional basal therapy, the control group treated with octreotide, while the observation group was treated with ulinastatin for 3-5 $\mathrm{d}$ in addition of the control group. Serum was used to measure the levels of liver function indicators, clinical efficacy, symptom improvement and complications before and after treatment in both groups. The results showed that the efficacy rate (significant rate+improvement rate) of the treatment group at least $5 \mathrm{~d}$ of treatment was $85.14 \%$, which was $61.61 \%$ higher than that of the control group; the time for abdominal distension relief, disappearance of pancreatic oedema, disappearance of abdominal pain, return of white blood cells to normal and return of amylase to normal after 3-5 d of treatment was shorter in the treatment group than that of the control group; the levels of transaminase and total bilirubin in the two groups were not statistically significant before treatment $(p>0.05) ; 3-5 \mathrm{~d}$ after treatment, the levels of transaminase and total bilirubin in the two groups were significantly lower than those before treatment $(\mathbf{p}<\mathbf{0 . 0 5})$ and compared with the control group, the levels of transaminase and total bilirubin in the treatment group were significantly lower $(\mathbf{p}<0.05)$. The combination of octreotide and ulinastatin treatment has a good therapeutic effect on patients with acute severe pancreatitis, helps to reduce the level of liver function indicators and the safety of the drugs is relatively high, so it is worth promoting and applying.
\end{abstract}

Key words: Acute severe pancreatitis, ustatin, orexin, liver function indicators, efficacy, safety

Acute Pancreatitis (AP), as a common clinical disease, has become one of the critical acute abdomens in Intensive Care Unit (ICU) due to its rapid progress, high incidence of complications and poor prognosis ${ }^{[1]}$. It has been reported that the clinical mortality rate of AP is $10 \% \sim 30 \%$ and the mortality rate is higher for patients with infection ${ }^{[2]}$. Early clinical symptoms of AP patients are lack of specificity and some patients may be accompanied by systemic inflammatory response syndrome (SIRS) and with the continuous development of the disease, multiple organ dysfunction syndrome (MODS), progression to multiple organ failure (MOF) and even threaten the lives of patients ${ }^{[3]}$.

The pathogenesis of AP is complex, which may be related to biliary obstruction, pancreatic duct obstruction, long term drinking, infection, pancreatic trauma and hyperlipidemia. At present, pancreatic enzyme activation and inflammatory response are the clinical research hotspots of AP treatment ${ }^{[4]}$. When AP occurs, multiple organ failure may occur if the disease is not controlled in time. Although clinical and experimental studies have made significant progress in pathophysiology and course development of severe acute pancreatitis, there is still a lack of understanding of the necrotizing inflammation of pancreas from glandular necrosis to microcirculation damage in pancreatic tissue $48-72 \mathrm{~h}$ after onset ${ }^{[5,6]}$. How to block the pathogenesis of the disease so as to block the progress of the disease has become the focus of clinical work and research. Therefore, blocking the activation of trypsin it, preventing and treating the generation and development of systemic inflammatory response are 
important measures and directions of treatment. The comprehensive treatment of severe acute pancreatitis (SAP) with protease inhibitors and inhibitors of cystine secretion is the current research hotspot. Octreotide, as an octapeptide somatostatin analogue of synthetic natural somatostatin, has many physiological activities such as long half-life and convenient use ${ }^{[7,8]}$. Ulinastatin is a trypsin inhibitor, which can effectively inhibit the secretion of trypsin and reduce the activity of trypsin ${ }^{[9]}$. Studies have shown that ulinastatin and octreotide for AP patients can help to reduce the level of inflammatory factors and improve the immune function of patients, but the test results are still controversial. Therefore, this study comprehensively studied the effects of ulinastatin and octreotide on inflammatory factors, immune cells and immunoglobulin, and compared the clinical effects. In addition, we also explored the combination of two drugs in the treatment of sap, blocking the inflammatory cascade reaction and prognosis, aiming to provide practical reference for the clinical application of ulinastatin and octreotide. A total of 224 AP patients were selected in our hospital from January 2017 to December 2019 as the subjects of this retrospective study and the subjects were diagnosed clearly according to the diagnostic guidelines. In addition, according to the severity of the disease, patients were divided into three types: medium acute pancreatitis (MAP), medium severe acute pancreatitis (MSAP), SAP. The subjects were randomly divided into control group $(n=112)$ and treatment group $(n=112)$. Both groups were treated with conventional basic therapy, the control group treated with octreotide, while the observation group was treated with ulinastatin. The control group included 76 males and 36 females, with an average age of $(52.16 \pm 8.23) \mathrm{y}$ (range, 22-88 y). According to the severity of AP, there were 106 cases of MAP+MSAP and 6 cases of SAP. There were 80 males and 32 females in the treatment

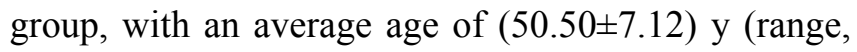
24-85) y. APACHE II score was (14-20) points, with an average of $(18.89 \pm 1.02)$ points. According to the severity of AP: MAP+MSAP $(n=111), \operatorname{SAP}(n=1)$. There was no significant difference in baseline data between the two groups $(\mathrm{p}>0.05)$. Inclusive criteria: male and female, aged $18-80 y$, in line with the clinical diagnostic criteria for acute pancreatitis in the guidelines for the diagnosis and treatment of acute pancreatitis. All patients were hospitalized within $24 \mathrm{~h}$ after the onset of clinical symptoms, without gastrointestinal cancer and severe autoimmune diseases; those who met the treatment indications of somatostatin and ulinastatin. Exclusion criteria: patients with obvious history of drug allergy in previous medical history or allergic to somatostatin and/ or ulinastatin in the treatment plan. Patients with onset time more than $24 \mathrm{~h}$, or those who died shortly after admission, or those with severe underlying diseases and dying state; patients with trauma, pregnancy, immunodeficiency disease or severe cardiovascular and cerebrovascular diseases.

Treatment efficiency (Significant effective +improvement): at least $5 \mathrm{~d}$ of continuous treatment, the treatment efficiency was evaluated from the perspectives of obvious effect, improvement and ineffectiveness. Significant effective: symptoms and signs disappear and normal life can be achieved. Improvement: symptoms and signs have improved, family members are needed to assist in life. Ineffective: the treatment plan is invalid or needs to be adjusted. Treatment efficiency=significant efficiency+improvement rate; Symptom improvement time: after 3-5 d of treatment, the time required for abdominal distension, pancreatic edema, abdominal pain, white blood cells and amylase in the two groups were recorded; Liver function indicators. The changes of liver function indexes transaminase and total bilirubin before and after treatment were recorded; Length and cost of hospitalization. Comparative analysis of the time and cost of the two groups of patients from hospitalization to discharge after diagnosis; Safety: after 3-5 d of treatment, the incidence of nausea and vomiting, liver function damage, hypoxemia, abdominal cavity and pancreatic cysts were counted. All data were processed by SPSS 19.0 software. The count data were analyzed by chi square test and expressed by $n(\%)$. The data were measured by (s) line. $\mathrm{p}<0.05$ was considered to be statistically significant. As shown in Table 1, after 3-5 d of treatment, the treatment efficiency (significant effective+improvement) of the treatment group was $85.14 \%$, significantly higher than $61.61 \%$ of the control group $(\mathrm{p}<0.05)$.

After 3-5 $\mathrm{d}$ of treatment, the time of abdominal distension, pancreatic edema, abdominal pain, white blood cells and amylase in the treatment group were shorter than those in the control group $(\mathrm{p}<0.05)$ (Table 2$)$, Pancreatitis occurs when the digestive enzymes

TABLE 1: COMPARISON OF TREATMENT EFFECT BETWEEN TWO GROUPS

\begin{tabular}{lccccccc}
\hline Group & $\mathbf{n}$ & Significant effective & Improvement & Ineffective & Effective rate & $\mathbf{X}^{2}$ & $\mathbf{p}$ \\
\hline Observation group & 112 & $68(60.71)$ & $28(25.00)$ & $16(14.29)$ & 85.14 & 5.467 & $<0.05$ \\
Control group & 112 & $52(46.43)$ & $17(15.18)$ & $43(38.39)$ & 61.61 & \\
\hline
\end{tabular}


www.ijpsonline.com

TABLE 2: COMPARISON OF SYMPTOM IMPROVEMENT BETWEEN TWO RESEARCH GROUPS

\begin{tabular}{|c|c|c|c|c|c|c|}
\hline Group & $\mathbf{n}$ & $\begin{array}{l}\text { Abdominal } \\
\text { remission }\end{array}$ & $\begin{array}{c}\text { Pancreatic edema } \\
\text { disappeared }\end{array}$ & $\begin{array}{c}\text { Abdominal pain } \\
\text { disappeared }\end{array}$ & $\begin{array}{c}\text { Recovery of white } \\
\text { blood cell count }\end{array}$ & Amylase \\
\hline Observation group & 112 & $7.29 \pm 1.29$ & $6.32 \pm 1.09$ & $4.32 \pm 1.06$ & $6.25 \pm 1.13$ & $6.54 \pm 1.37$ \\
\hline Control group & 112 & $11.26 \pm 1.45$ & $9.66 \pm 1.33$ & $9.25 \pm 1.24$ & $12.45 \pm 1.29$ & $12.52 \pm 1.67$ \\
\hline $\mathrm{t}$ & & 12.163 & 11.724 & 13.671 & 15.034 & 15.732 \\
\hline $\mathrm{p}$ & & $<0.001$ & $<0.001$ & $<0.001$ & $<0.001$ & $<0.001$ \\
\hline
\end{tabular}

TABLE 3: CHANGES OF THE LIVER FUNCTION INDICATORS OF THE TWO RESEARCH GROUPS

\begin{tabular}{lccccc}
\hline \multirow{2}{*}{ Group } & $\mathrm{n}$ & \multicolumn{2}{c}{ Before treatment } & \multicolumn{2}{c}{ After treatment } \\
\cline { 3 - 6 } & & Transaminase $(\mu \mathrm{mol} / \mathrm{l})$ & Total bilirubin $(\mu \mathrm{mol} / \mathrm{l})$ & Transaminase $(\mu \mathrm{mol} / \mathrm{l})$ & Total bilirubin $(\mu \mathrm{mol} / \mathrm{l})$ \\
Observation group & 112 & $124.30 \pm 16.87$ & $43.27 \pm 10.31$ & $49.86 \pm 15.13$ & $16.56 \pm 4.54$ \\
Control group & 112 & $125.07 \pm 17.28$ & $44.52 \pm 10.46$ & $81.24 \pm 22.33$ & $20.27 \pm 6.89$ \\
$\mathrm{t}$ & & 1.082 & 1.723 & 9.206 & 6.237 \\
$\mathrm{p}$ & 0.945 & 0.787 & $<0.001$ & 0.002 \\
\hline
\end{tabular}

primarily trypsin are activated within the pancreas and the end result is autodigestion of the pancreas and surrounding tissues. The levels of transaminase and total bilirubin before treatment in the two groups were not statistically significant $(\mathrm{p}>0.05)$; 3-5 d after treatment, the levels of transaminase and total bilirubin in the two groups were significantly lower than those before treatment $(p<0.05)$ and compared with the control group, the levels of transaminase and total bilirubin in the treatment group were significantly lower $(\mathrm{p}<0.05)$, as shown in Table 3. After 3-5 d of treatment, the results showed that the hospital stay of the treatment group was significantly lower than that of the control group. AP, with the characteristics of acute onset, rapid changes in the condition and high incidence of complications, is a common critical and severe clinical disease ${ }^{[10]}$ and the clinical mortality rate reaches $10 \%-30 \%{ }^{[11]}$. In the early stage of the disease, patients can have shock, respiratory dysfunction, renal dysfunction and other complications, threatening the lives of patients. Although AP is a multifactorial and complex pathological process, it will eventually lead to local and/or systemic inflammatory reactions. In the pathogenesis of severe AP patients, systemic inflammatory response and systemic infection becomes the peak of death in AP patients ${ }^{[12]}$. Therefore, inflammation throughout the development of AP will aggravate the occurrence and development of the disease $^{[13]}$. Ulinastatin is mainly a glycoprotein purified from male urine, composed of about 143 amino acids, and is a broad spectrum protease inhibitor ${ }^{[14]}$. It has inhibitory effects on trypsin, granulocyte elastase, plasmin and many other enzymes; in addition, it has a relatively stable lysosomal membrane, which can inhibit the release of lysosomal enzymes and effectively remove oxygen free radicals ${ }^{[15]}$. Octreotide has an inhibitory effect on the secretion of gastric acid, pancreatic enzymes, glucagon and insulin. It can reduce gastric motility, gallbladder emptying, inhibits mucosal epithelial cell necrosis, enhance intestinal cell activity, reduce intestinal endotoxins, bacteria translocation, prevent the release of inflammatory factors, and improve the body's immunity ${ }^{[16]}$. The results of this study showed that the effective rate of clinical treatment in the observation group after treatment was significantly higher than that in the control group and the liver function indicators in the observation group were significantly lower than those in the control group $(p<0.05)$, suggesting that ulinastatin combined with octreotide treatment ASP has a significant clinical effect and can effectively improve liver function. The reason is that ulinastatin can inhibit a variety of proteases and reduce the damage caused by oxygen free radicals to multiple organs in patients. In addition, the combination of ulinastatin and octreotide is safer in AP patients. In this study, after 3-5 d of treatment, the treatment efficiency of the treatment group was $85.14 \%$, significantly higher than $61.61 \%$ of the control group, indicating that the combined use of ulinastatin and octreotide can improve the clinical treatment efficiency in the near future (3-5 d) and promote the early recovery of patients. Some studies have found that ulinastatin can inhibit multiple pathological and physiological processes of AP, stabilize the biological activities of various enzymes in the cell membrane of the body, prevent intracellular calcium overload, so as to improve local blood perfusion, shorten the time for symptom improvement and promote the recovery of patients ${ }^{[17,18]}$. In this study, after 3-5 d of treatment, the time of abdominal distension, pancreatic edema, abdominal pain, white blood cells and amylase in the treatment group were shorter than those in the control 
group, suggesting that ulinastatin can shorten the time for symptom improvement and promote the early recovery of patients. In conclusion, the combination of octreotide and ulinastatin treatment has ideal short term effect for AP patients, which helps to improve the treatment efficiency, shorten the time for symptom improvement and the drug safety is relatively high, which is worthy of promotion and application. On the other hand, this study also revealed that intravenous administration of ulinastatin reduced mortality in patients with severe sepsis.

\section{Conflicts of interest:}

The authors report no conflicts of interest.

\section{Acknowledgements:}

This work was supported by the Beijing Luhe Hospital Captial Medical Univeisity.

\section{REFERENCES}

1. Bank S, Singh P, Pooran N, Stark B. Evaluation of factors that have reduced mortality from acute pancreatitis over the past 20 years. J Clin Gastroenterol 2002;35:50-60.

2. Wang G, Liu Y, Zhou SF, Qiu P, Xu L, Wen P, et al. Effect of somatostatin, ulinastatin and gabexate on the treatment of severe acute pancreatitis. Am J Med Sci 2016;351:506-12.

3. Skálová A, Weinreb I, Hyrcza M, Simpson RH, Laco J, Agaimy A, et al. Clear cell myoepithelial carcinoma of salivary glands showing EWSR1 rearrangement: molecular analysis of 94 salivary gland carcinomas with prominent clear cell component. Am J Surg Pathol 2015;39:338-48.

4. Wang X, Zhuang X, Wei R, Wang C, Xue X, Mao L. Protective effects of Acanthopanax vs. Ulinastatin against severe acute pancreatitis-induced brain injury in rats. Int Immunopharmacol 2015;24:285-98.

5. Luo Y, Che W, Zhao M. Ulinastatin post-treatment attenuates lipopolysaccharide-induced acute lung injury in rats and human alveolar epithelial cells. Int J Mol Med 2017;39:297306.

6. Abreu FF, Souza AC, Teixeira SA, Soares AG, Teixeira DF, Soares RC, et al. Elucidating the role of oxidative stress in the therapeutic effect of rutin on experimental acute pancreatitis. Free Radic Res 2016;50:1350-60.

7. Chen KL, Lv ZY, Yang HW, Liu Y, Long FW, Zhou B, et al. Effects of tocilizumab on experimental severe acute pancreatitis and associated acute lung injury. Crit Care Med 2016;44:e664-77.
8. Huang EC, Ghazikhanian V, Qian X. Giant cell-rich osteosarcoma of the parotid gland: An exceptionally rare entity at an unusual site. Diagn Cytopathol 2016;44:1107-11.

9. Li W, Qiu X, Jiang H, Zhi Y, Fu J, Liu J. Ulinastatin inhibits the inflammation of LPS-induced acute lung injury in mice via regulation of AMPK/NF- $\kappa$ B pathway. Int Immunopharmacol 2015;29:560-7.

10. Wan X, Xie X, Gendoo Y, Chen X, Ji X, Cao C. Ulinastatin administration is associated with a lower incidence of acute kidney injury after cardiac surgery: a propensity score matched study. Crit Care 2016;20:1-7.

11. Thomsen RW, Pedersen L, Møller N, Kahlert J, Beck-Nielsen $\mathrm{H}$, Sørensen HT. Incretin-based therapy and risk of acute pancreatitis: a nationwide population-based case-control study. Diabetes Care 2015;38:1089-98.

12. Bettaieb A, Chahed S, Bachaalany S, Griffey S, Hammock BD, Haj FG. Soluble epoxide hydrolase pharmacological inhibition ameliorates experimental acute pancreatitis in mice. Mol pharmacol 2015;88:281-90.

13. Chang CC, Chiou CS, Lin HL, Wang LH, Chang YS, Lin HC. Increased risk of acute pancreatitis in patients with rheumatoid arthritis: A population-based cohort study. PLoS One 2015; 10:e0135187.

14. Ju M, He H, Chen S, Liu Y, Liu Y, Pan S, et al. Ulinastatin ameliorates LPS-induced pulmonary inflammation and injury by blocking the MAPK/NF- $\mathrm{KB}$ signaling pathways in rats. Mol Med Rep 2019;20:3347-54.

15. Meng L, Wu Z, Zhang H. Effect of ulinastatin combined with octreotide on serum endothelin, endotoxin levels and immune function in acute pancreatitis. J Coll Physicians Surg Pak 2019;29:90-2.

16. Wang R, Yang F, Wu H, Wang Y, Huang Z, Hu B, et al. High-dose versus low-dose octreotide in the treatment of acute pancreatitis: a randomized controlled trial. Peptides. 2013;40:57-64.

17. Honore PM, Spapen HD. Ulinastatin to prevent acute kidney injury after cardiopulmonary bypass surgery: does serum creatinine tell the whole story?. Crit Care 2016;20:1-2.

18. Bonnet U, Taazimi B, Montag M, Ronge R, Gespers H, Kuhlmann $\mathrm{R}$, et al. Severe acute pancreatitis, neuroleptic malignant syndrome and grand mal seizures associated with elevated amisulpride and low clozapine serum levels. Psychiatr Danub 2015;27:0-425.

This is an open access article distributed under the terms of the Creative Commons Attribution-NonCommercial-ShareAlike 3.0 License, which allows others to remix, tweak, and build upon the work non-commercially, as long as the author is credited and the new creations are licensed under the identical terms

This article was originally published in a special issue, "Clinical Research in Pharmaceutical and Biomedical Sciences" Indian J Pharm Sci 2021:83(1)Spl issue1;75-78 